\title{
Buildup of the ring current during periodic loading-unloading cycles in the magnetotail driven by steady southward interplanetary magnetic field
}

\author{
A. Taktakishvili, ${ }^{1}$ M. M. Kuznetsova, ${ }^{1}$ M. Hesse,${ }^{1}$ M.-C. Fok, ${ }^{1}$ L. Rastätter, ${ }^{1}$ M. Maddox,${ }^{1}$
} A. Chulaki, ${ }^{1}$ T. I. Gombosi, ${ }^{2}$ and D. L. De Zeeuw ${ }^{2}$

Received 1 February 2007; revised 1 May 2007; accepted 11 June 2007; published 8 September 2007.

[1] During prolonged intervals of negative interplanetary magnetic field (IMF) $B_{z}$ the magnetosphere often enters a state in which quasi-periodic, large-amplitude oscillations of energetic particle fluxes are observed at the geosynchronous orbit. We use the global magnetosphere MHD code BATS-R-US output during a long period of steady southward IMF $B_{z}$ to drive the Fok Ring Current Model. Previous simulations of such events demonstrated flat behavior of the energetic particle fluxes after the initial injection. Periodical north/south IMF turning was required to reproduce oscillations in particle fluxes at geosynchronous orbit. In the present study we use a global magnetosphere MHD code that reproduces fast magnetotail reconnection rates observed in kinetic simulations. This results in periodical loading-unloading cycles in the magnetotail even for steady southward $B_{z}$ and can explain quasi-periodic oscillations of geosynchronous energetic particle fluxes. The total proton energy within geosynchronous orbit exhibits overall growth in time due to quasi-steady convection and oscillates due to injection through inductive electric field caused by multiple dipolarization. The flux oscillation amplitude is stronger in the outer regions of the ring current although the regions close to the geosynchronous orbit experience substantial perturbations as well.

Citation: Taktakishvili, A., M. M. Kuznetsova, M. Hesse, M.-C. Fok, L. Rastätter, M. Maddox, A. Chulaki, T. I. Gombosi, and D. L. De Zeeuw (2007), Buildup of the ring current during periodic loading-unloading cycles in the magnetotail driven by steady southward interplanetary magnetic field, J. Geophys. Res., 112, A09203, doi:10.1029/2007JA012317.

\section{Introduction}

[2] The Earth's ring current is a large-scale electric current system encircling Earth's magnetic equator at radial distances typically in the range from 2 to $8 R_{E}$ [e.g., Fok et $a l ., 2001]$. It is a population of hot electrons and ions with energy ranging from 1 to $300 \mathrm{keV}$ and density range $0.1-$ $10 \mathrm{~cm}^{-3}$. The current is carried mainly by westward drifting trapped ions [Daglis et al., 1999].

[3] The main source of particles of the ring current is the plasma sheet. The distribution of particles in the plasma sheet has a strong impact on the ring current dynamics [see, e.g., Ebihara et al., 2005]. During geospace storms particles are injected and accelerated from the plasma sheet into the ring current through the nightside geosynchronous orbit region [Wolf et al., 1997; Fok et al., 1996]. However, plasma sheet itself is directly influenced by the processes in the magnetotail, as was demonstrated for example by Fok

\footnotetext{
${ }^{1}$ Space Weather Laboratory, NASA Goddard Space Flight Center, Greenbelt, Maryland, USA.

${ }^{2}$ Center for Space Environment Modeling, University of Michigan, Ann Arbor, Michigan, USA.
}

Copyright 2007 by the American Geophysical Union. 0148-0227/07/2007JA012317 et al. [1999], where authors studied the evolution of the inner plasma sheet and ring current during substorms. All these and other studies indicate on a strong coupling between the magnetotail dynamics and the ring current buildup.

[4] The important questions, "what is the major mechanism of the ring current particle energization," and "what is the source population of the newly built ring current," are not completely solved yet. Some studies [Wolf and Harel., 1979; Wolf et al., 1997; Ebihara and Ejiri, 2000] consider the large-scale electric field driven by a period of prolonged southward IMF as a major cause of the ring current buildup. This electric field results in a global convection and subsequent particle injection into the inner magnetosphere. Other studies [Fok and Moore, 1997; Fok et al., 1999] point to the importance of substorms in ring current formation. Substorms can contribute significantly to the growth of the ring current through dipolarization of the magnetic field, as the dipolarization produces an inductive electric field that energizes particles [Birn et al., 2004a, 2004b].

[5] Recently, Keller et al. [2005], using a combination of the University of Michigan's global magnetosphere MHD BATS-R-US Model [Powell et al., 1999] and Fok Ring Current Model (FRC) [Fok et al., 1999; Fok and Moore, 1997], examined the effect of multiple substorms on the ring current. Multiple dipolarizations in the tail were modeled 
using periodic flipping (changing sign) of the IMF $B_{z}$. They found that ionospheric potential increases during periods of southward IMF $B_{z}$ and that energy growths are more dependent on the duration of large ionospheric potential than on the number of substorm dipolarizations. Since numerical resistivity alone produces a steady magnetosphere for steady solar wind conditions, periodical flipping of the IMF $B_{z}$ component was required to reproduce geosynchronous flux oscillations. Without the flipping of IMF $B_{z}$ the fluxes were basically flat. However, observations [e.g., see, Reeves and Henderson, 2001; Henderson et al., 2006a, 2006b] demonstrate that quasi-periodic, largeamplitude oscillations of energetic particle fluxes are often detected at the geosynchronous orbit during prolonged intervals of steady southward IMF $B_{z}$.

[6] Recently, Kuznetsova et al. [2006] used the BATS-RUS model to analyze the influence of different dissipation mechanisms triggering magnetic reconnection, including nongyrotropic effects, in the magnetotail region. The detailed description of their approach is given in the paper by Kuznetsova et al. [2007]. By introducing kinetic corrections, they were able to reproduce fast magnetotail reconnection rates observed in kinetic simulations and obtained quasi-periodic loading-unloading cycles (multiple reconnection) in the magnetotail even for steady southward IMF conditions.

[7] In the presented work we use the FRC model to investigate the buildup of the ring current during the magnetotail loading-unloading cycles in the coarse of the long period of steady southward IMF. As input to the FRC model we use the results of the simulation employing the technique developed by Kuznetsova et al. [2007]. This coupled modeling allowed us to reproduce, for the first time in theoretical simulations, the "sawtooth" oscillations in the ring current: quasi-periodic, large-amplitude oscillations of energetic particle fluxes at the geosynchronous orbit during prolonged intervals of negative IMF $B_{z}$.

\section{Brief Description of the Models Used}

[8] The University of Michigan global magnetosphere MHD model BATS-R-US [Powell et al., 1999] uses solar wind input as an upstream boundary condition. It calculates, self-consistently, a magnetic field, ionospheric potential, and plasma sheet temperature and density distributions that are then used as input to the FRC model. The BATS-R-US adaptive grid structure permits to increase resolution where and when it is needed, which in its turn makes possible to perform global simulations with spatial resolution comparable to ion kinetic scales. This allowed Kuznetsova et al. [2007] to include kinetic corrections to the MHD equations, taking into account nongyrotropy of ion motion near reconnection sites in the magnetotail region. As a result, Kuznetsova et al. [2007] obtained multiple, quasi-periodic reconnection in the magnetotail for steady southward IMF $B_{z}$ conditions, the result never accomplished before in theoretical simulations.

[9] This simulation make possible to obtain more physical dynamics of the magnetic reconnection in the magnetotail. Unphysical numerical resistivity alone produces a steady magnetosphere for steady solar wind conditions. That is why in previous simulations by Keller et al. [2005], periodical flipping of the IMF $B_{z}$ component was required to reproduce geosynchronous flux oscillations. On the other hand, the simulation by Kuznetsova et al. [2007] opened the way for modeling of the "sawtooth" events: it reproduces dynamical tail for steady solar wind with prolonged interval of southward IMF $B_{z}$. For more detailed description of their simulation, see the paper by Kuznetsova et al. [2007].

[10] The Fok ring current model (FRC) [Fok et al., 1999; Fok and Moore, 1997] calculates the differential particle fluxes for protons and electrons up to $300 \mathrm{keV}$ by solving a bounce-averaged Boltzmann transport equation for a phase space distribution function along magnetic field lines. The phase space distribution is assumed to be constant along magnetic field lines. The advection terms include gradient curvature drift and $\mathbf{E} \times \mathbf{B}$ drift, which includes both corotation and the convection. In addition, the model calculates losses due to charge exchange. The initial source population uses the quiet time ion composition compiled by Sheldon and Hamilton [1993], which was obtained using Active Magnetospheric Particle Tracer Explorer/Charge Composition Explorer/Charge-Energy-Mass instrument. However, the initial composition is not that important for our analysis because of the "losing of the memory" of the initial distribution, due to the charge-exchange losses, driftout of the particles on the growth phase of the substorm, and later inflowing of the energized particles accelerated during the loading-unloading cycles in the magnetotail. After the initial setup, FRC uses as an input temperature and density of protons at the outer boundary of the model $\left(10 R_{E}\right.$ on the nightside and the last closed field line on the dayside) and ionospheric potential and magnetic field calculated by MHD code BATS-R-US. The pitch angle distribution on the boundary is assumed to be isotropic.

\section{Solar Wind Input Conditions}

[11] The solar wind parameters, velocity $V_{x}=-500 \mathrm{~km} / \mathrm{s}$, $V_{y}=V_{z}=0$, density $n=5 \mathrm{~cm}^{-3}$, temperature $T=2 \cdot 10^{5} \mathrm{~K}$, and IMF magnetic field $\mathrm{x}$ and y components $B_{x}=B_{y}=0$, remain the same throughout the whole analysis. For IMF $B_{z}$ component we consider the following scenario: at the start (00h:00m) $B_{z}$ is southward $B_{z}=-15 \mathrm{nT}$, then at 01:05 turns northward $B_{z}=15 \mathrm{nT}$ and stays this way for 3 hours until 04:05. At 04:05 $B_{z}$ changes sign again back to southward $B_{z}=-15 \mathrm{nT}$ and remains southward for the rest of the simulations. This behavior of IMF $B_{z}$ is shown in Figure 1 (the thin solid line). The cross polar cap potential $\Phi$, calculated from the BATS-R-US model ionosphere part is also shown in the same plot. There is a characteristic drop of $\Phi$ for northward IMF $B_{z}$ component and rapid growth as IMF $B_{z}$ changes its sign to southward direction.

[12] The split of the curve for $\Phi$ after 04:30 in Figure 1 corresponds to different simulation conditions considered by Kuznetsova et al. [2007]. The solid line represents the simulations when gyrotropic effects are taken into account. The oscillatory behavior of $\Phi$ in this case is due to the periodic loading-unloading cycles or, in other words, multiple reconnection in the magnetotail. The dotted line on the contrary corresponds to the case when no gyrotropic effects are considered and only steady reconnection takes place. 


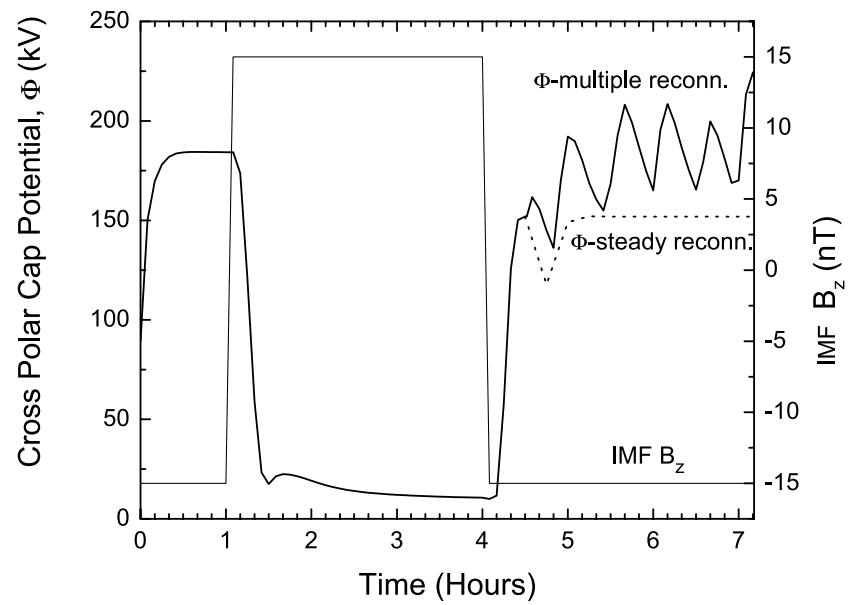

Figure 1. The input interplanetary magnetic field (IMF) $B_{z}$ component (thin line) and the output for the cross polar cap potential (bold line) given by the BATS-R-US code during the whole simulation period.

Cross polar cap potential $\Phi$ demonstrates flat behavior in this case.

\section{Simulation Results}

[13] Here we present results of ring current buildup when nongyrotropy effects in the BATS-R-US global magnetosphere MHD simulation were taken into account. We consider only the $\mathrm{H}^{+}$ion (proton) fluxes in our analysis.

[14] In Figure 2 by bold solid line is shown $B_{z}$ field component time variation at the midnight MLT (MLT = 00:00) point of the geosynchronous orbit $\mathrm{R}=6.6 R_{E}$ and $120 \mathrm{keV}$ proton flux (dotted line) at the same location calculated using FRC model. Also plotted (thin solid line) for reference is IMF $B_{z}$. As shown in Figure 2 the particle flux exhibits large amplitude oscillations after $B_{z}$ turned southward at 04:05. We will concentrate mostly on this part of the simulation interval, since we are interested in the ring current buildup during periodic loading-unloading in the magnetotail.

[15] The clear correlation between tail magnetic field and flux oscillations indicates that the cause of flux oscillations is bursty injection of energetic particles from the tail. Particles are energized by the inductive electric field generated during magnetic field variation in the course of the periodic loading-unloading in the magnetotail. Therefore in contrast to the result of Keller et al. [2005], geosynchronous flux oscillations are not necessarily caused by IMF $B_{z}$ flipping/cross polar cap potential variations. The drop of the flux at a little bit earlier than 05:00, following the 3 hour quiet period of northward IMF $B_{z}$, is a characteristic "growth phase dropout" caused by the deenergization of the ring current particles on the growth phase of the substorm: southward turn of IMF $B_{z}$ at 04:05 causes tailward stretching of the Earth's magnetic field and particle energy drop due to the conservation of the third adiabatic invariant.

[16] At about 05:00 the first burst of particle flux is observed. This peak value corresponds to the injection of particles energized during the first reconnection event that

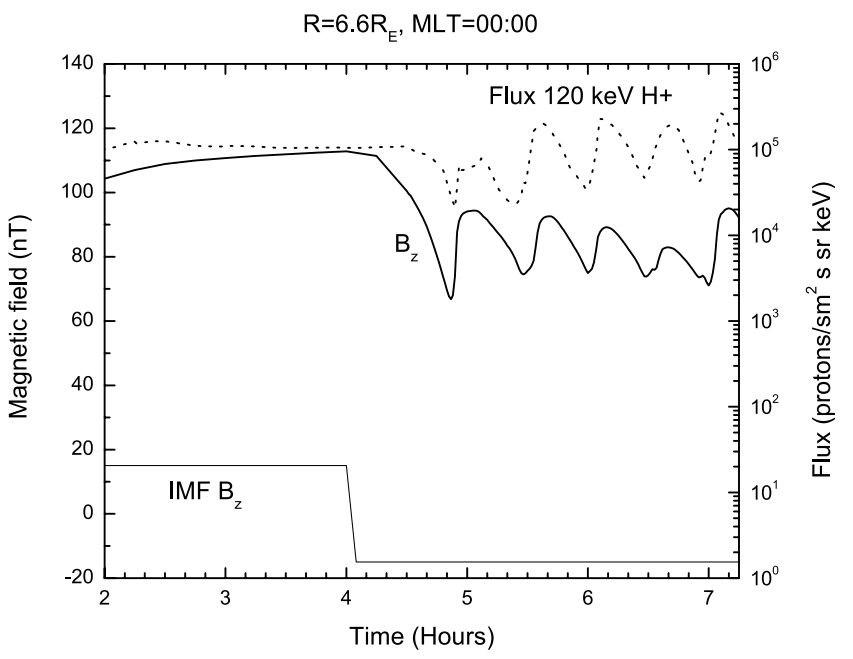

Figure 2. $B_{z}$ field component (bold solid line) given by the BATS-R-US code and the output of the FRC model for $120 \mathrm{keV}$ proton flux (dotted line) at the geosynchronous orbit $R=6.6 R_{E}, \mathrm{MLT}=00: 00$. Thin solid line corresponds to the input IMF $B_{z}$ component.

occurred at approximately 04:52. The second unloading happened at approximately 05:34 and it echoed in the second peak value of flux at approximately $05: 40$. There are three more peaks in the flux with the correspondent oscillations of $B_{z}$ field. The simulation stopped soon after the fifth and the last unloading occurred at approximately 07:00. The calculations clearly demonstrate strong oscillatory behavior of the energetic particle fluxes even for the steady southward IMF $B_{z}$ conditions.

[17] Figure 3 shows the time series of the geosynchronous proton flux for three energies, 60,120 , and $240 \mathrm{keV}$ at
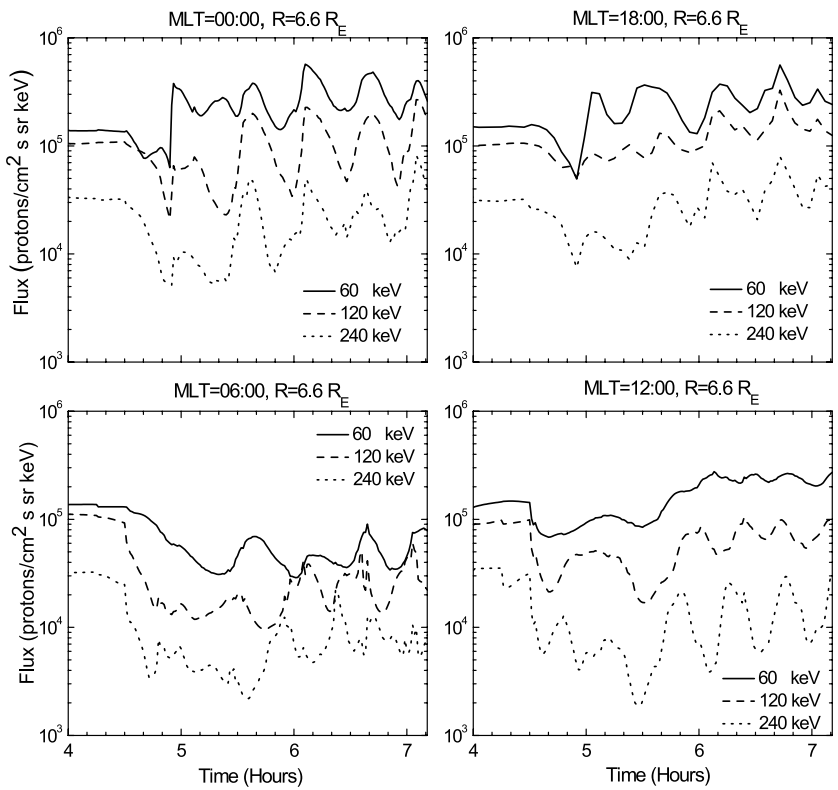

Figure 3. The FRC model output for the geosynchronous orbit proton fluxes for three different energy channels, $60 \mathrm{keV}$ (solid line), $120 \mathrm{keV}$ (dashed line) and 240 (dotted line) $\mathrm{keV}$, and four different MLT positions. 

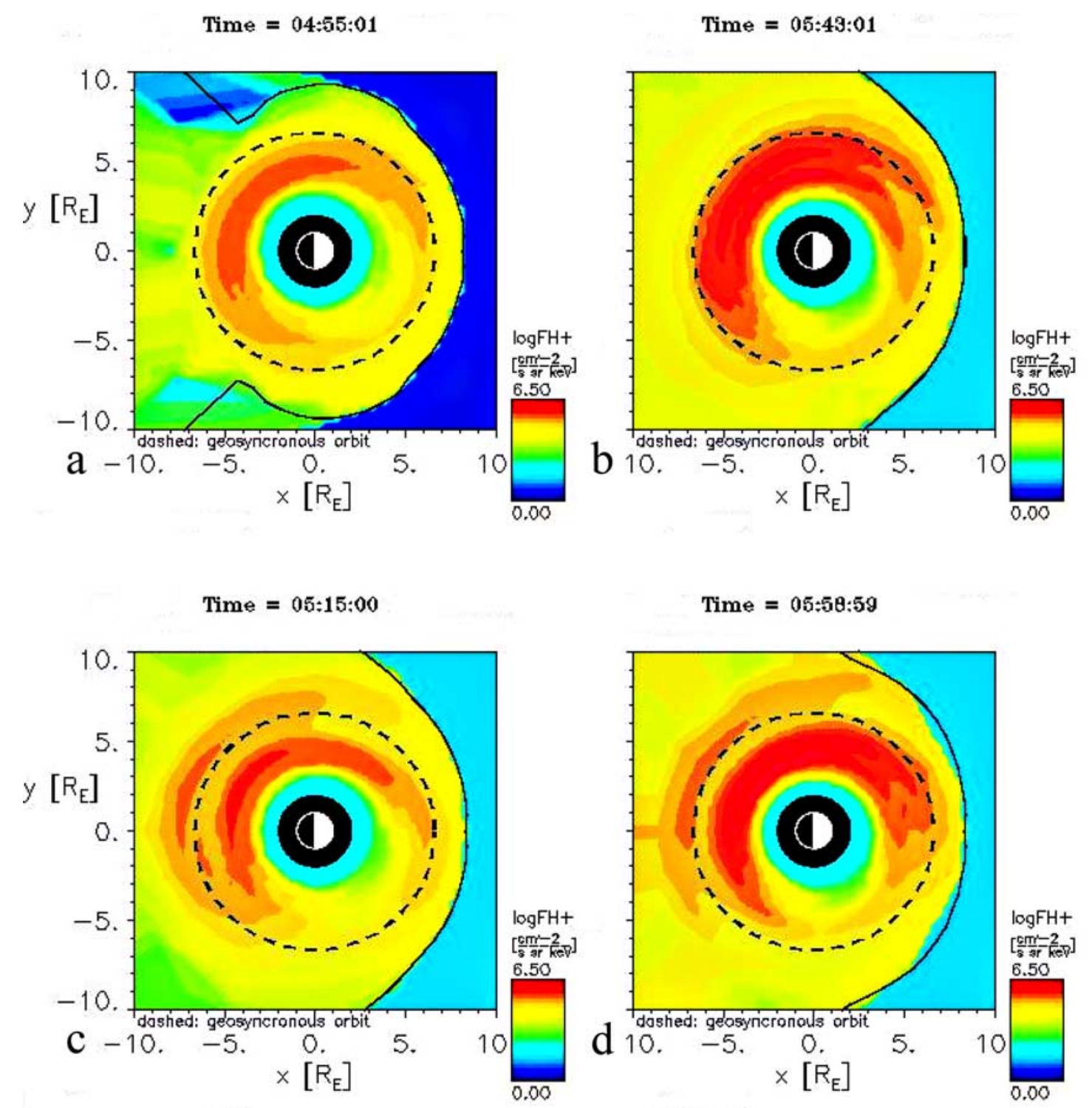

Figure 4. Color-coded $60 \mathrm{keV}$ proton fluxes in equatorial plane for different time stamps of the FRC model simulation. The scale is logarithmic. Solid lines correspond to FRC model boundary and dashed lines correspond to geosynchronous orbit. Shown are (a) the flux just few minutes after the first reconnection occurred in the magnetotail at approximately 04:52, before the particles accelerated in the magnetotail reached the ring current region. (c) The read crescent between 00:00 and 21:00 MLT, just outside the geosynchronous orbit demonstrates the injection of particles taking place later at 05:15. Also shown are (b) the flux few minutes after the second reconnection occurred at approximately 05:34 and (d) the following injection of particles into the ring current.

different MLT. All fluxes exhibit strong oscillations. At MLT 00:00 fluxes are almost dispersionless: peaks are reached simultaneously. When going in westward direction (clockwise in this plot) fluxes exhibit increasing energy dispersion: more energetic particles are detected first followed by the lower-energy particles, since westward gradient/curvature drift velocity of particles is proportional to their energy.

[18] Figure 4 demonstrates $60 \mathrm{keV}$ energy proton equatorial flux snapshots after the first (Figures $4 \mathrm{a}$ and $4 \mathrm{c}$ ) and the second (Figures $4 \mathrm{~b}$ and $4 \mathrm{~d}$ ) reconnection events in the tail. Solid lines correspond to the FRC model boundary and dashed lines correspond to the geosynchronous orbit. Figure 4a shows the flux at 04:55, just a few minutes after the first reconnection occurred in the magnetotail at approximately 04:52 but before the particles accelerated in the magnetotail reached the ring current region. The partial ring current formed by the preexisting particles, before the first injection occurred, is indicated by the red crescent inside the geosynchronous orbit. Figure 4c shows the arrival at $05: 15$ of the newly injected particles, indicated by the red crescent just outside the geosynchronous orbit, between $\sim 00: 00$ and $\sim 21: 00$ MLT. The double pick structure of the $60 \mathrm{keV}$ particle spatial distribution in Figure $4 \mathrm{c}$ is a manifestation of the coexistence of two separate populations in the ring current. The energization of the preexisting ring current 

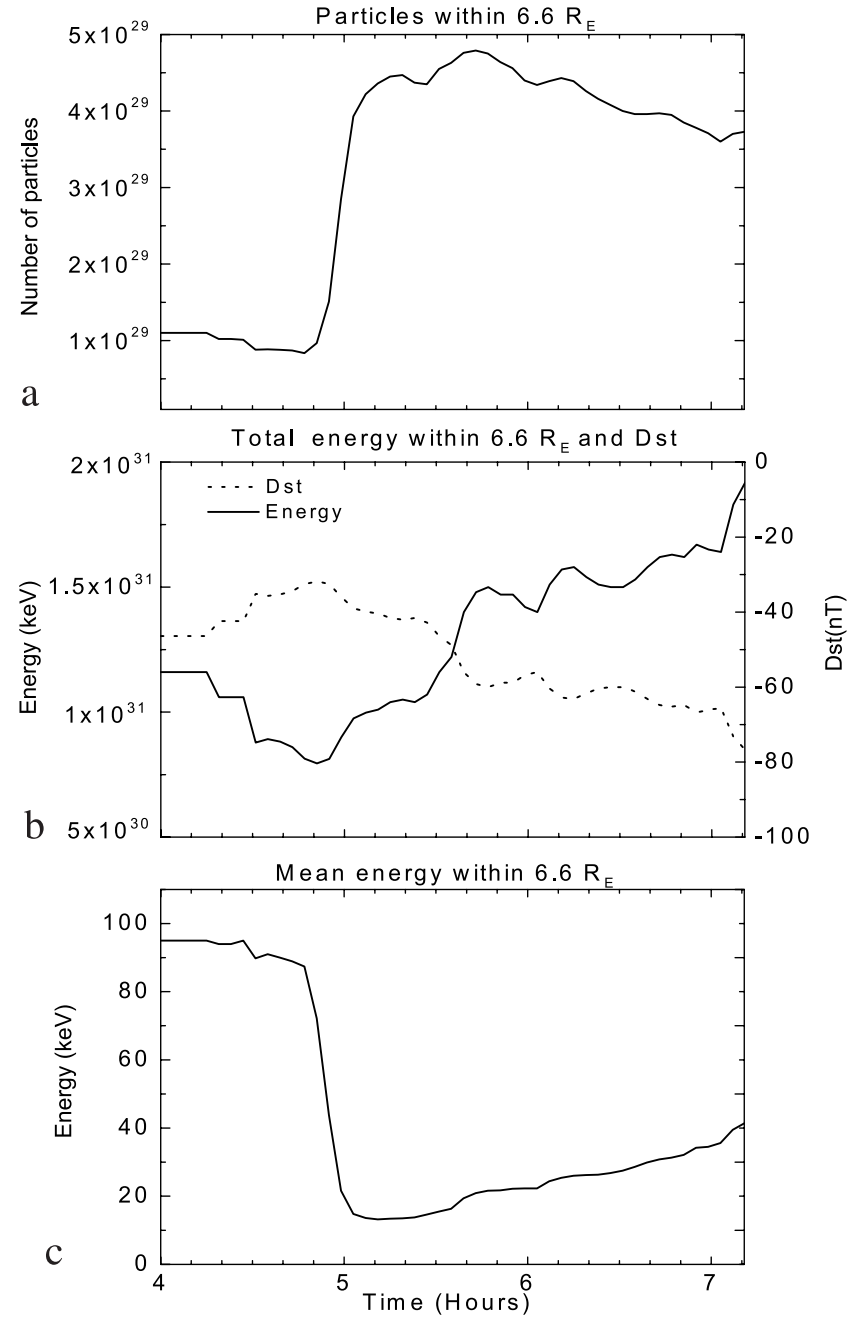

Figure 5. The output of the FRC model for (a) the time evolution of the total number of protons within geosynchronous orbit, (b) their total energy within the geosynchronous orbit and correspondent Dst index variation, and (c) particle mean energy.

population inside the geosynchronous orbit, indicated by the enhancement of the red color, is due to the inductive electric field associated to the dipolarization of the magnetic field in the course of the substorm. Figure $4 \mathrm{~b}$ shows these two particle populations merged and the expanding enhanced region. The time stamp of Figure $4 \mathrm{~b}$ is $05: 43$, several minutes after the second reconnection occurred at approximately $05: 34$. Figure $4 d$ shows the arrival of the accelerated particles at 05:59, indicated by the red crescent just outside the geosynchronous orbit, between $\sim 00: 00$ and $\sim 22: 00$ MLT. The ring current becomes relatively symmetrical.

[19] The Fok ring current model allows to calculate particle number and energy content in different regions. Figure 5a shows the number of protons within geosynchronous orbit as a function of time. There is a sharp increase after the first reconnection event at 4:52. The growth continues after the second reconnection event and is followed by a slow decrease with superimposed weak oscillations due to the consequent loading-unloading cycles in the tail. The high level of the number of particles within geosynchronous orbit is maintained by quasi-steady convection into the ring current for steady southward IMF $B_{z}$ component. The slow decrease is caused by the increasing losses due to charge exchange and drift loss at the dayside magnetopause. Therefore we obtained overall growth of the number of particles in the geosynchronous region in contrast to the result of Keller et al. [2005] where authors obtained rather strong oscillations but no overall growth of the number of particles within geosynchronous orbit due to IMF $B_{z}$ flipping/cross polar cap potential variations.

[20] Figure 5b shows the time evolution of the total proton energy within geosynchronous orbit, i.e., within the ring current. In Figure $5 \mathrm{~b}$ we show correspondent Dst index variation, produced by the simulated ring current and estimated using Dessler-Parker-Skopke relation [Dessler and Parker, 1959]. Dst reaches its maximum value of approximately $-80 \mathrm{nT}$ at the end of the simulation period, indicating that the simulated storm is of a moderate strength. After the IMF $B_{z}$ turned southward at 04:05, the total energy within geosynchronous orbit starts to drop in respect to its quiet time (presubstorm) level. This corresponds to the growth phase of the substorm, when due to the stretching of the magnetosphere particles are drifting out of the inner regions. After the first reconnection occurs at 05:52 the energy starts to grow again due to quasi-steady convection with superimposed oscillations due to the reconnection events. At approximately 05:30 it exceeds its presubstorm level which is a clear indication of the storm and substorm contributions to the ring current energy. The combined action of quasi-steady convective and bursty inductive electric fields manifested in overall growth with superimposed oscillations of the total energy is in agreement with observations of the ring current during "sawtooth"

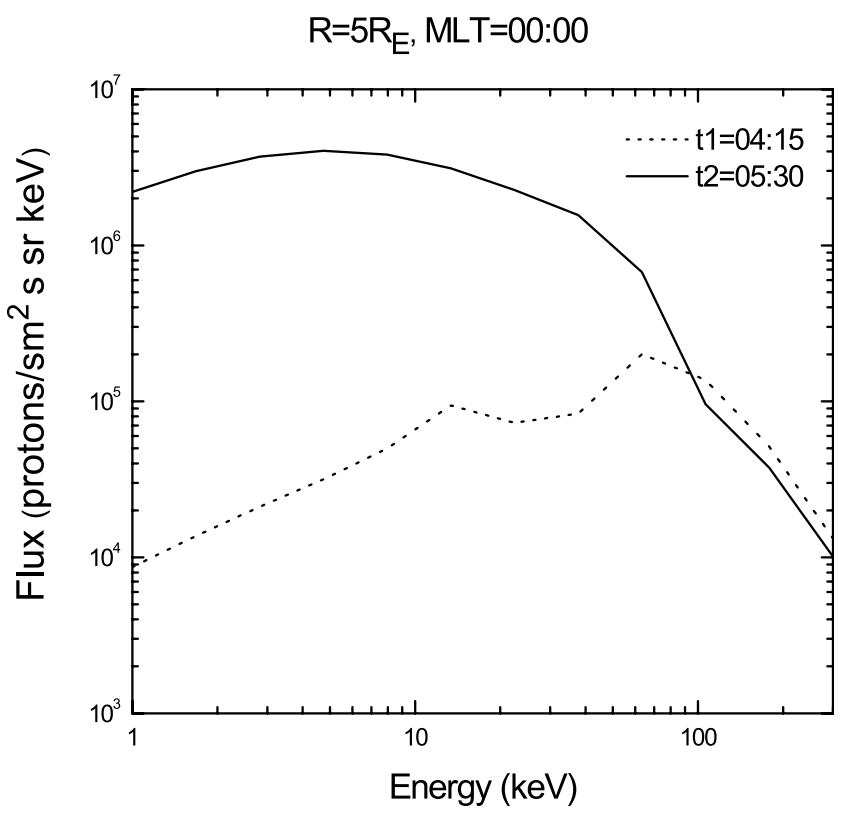

Figure 6. The spectra of particles for two different moments of time: before and after the first reconnection occurred at 04:52. The dotted line represents the spectrum of particles at the presubstorm moment of time $t 1=04: 15$. The solid line in Figure 6 shows the spectrum of particles at the postsubstorm moment of time $\mathrm{t} 2=05: 30$. 
Particle injection and loss through the boundaries

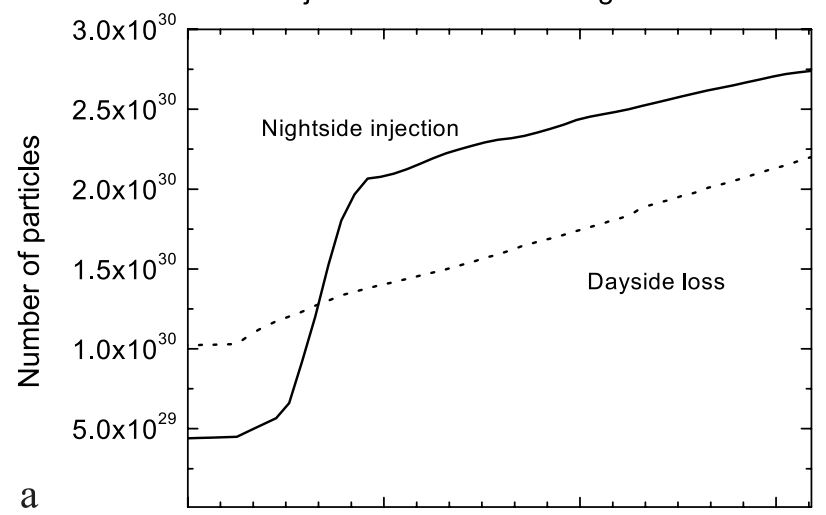

Energy gain and loss through the boundaries

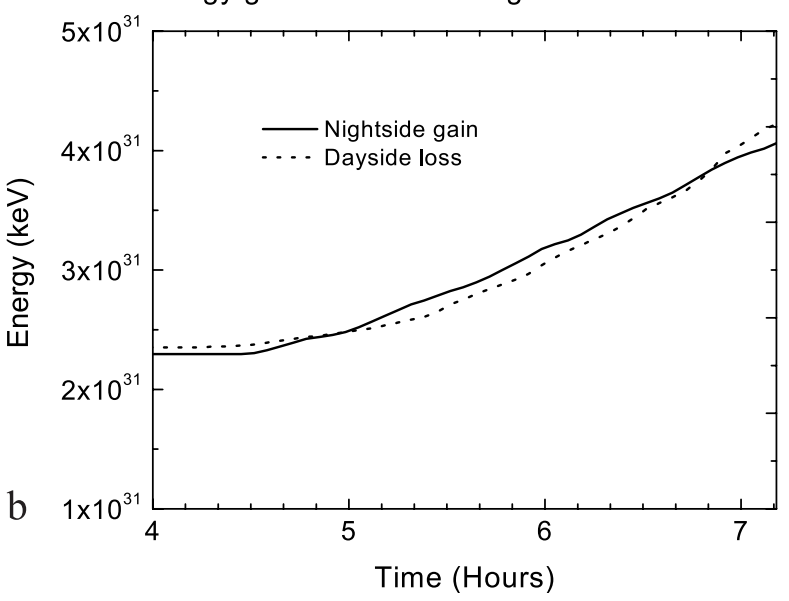

Figure 7. (a) Proton number and (b) energy gain and loss through the FRC model nightside and dayside boundaries as a function of time.

geosynchronous injections [Reeves et al., 2004]. In comparison, simulations by Keller et al. [2005] demonstrated no overall growth of the ring current energy due to IMF $B_{z}$ flipping/cross polar cap potential variations.

[21] Figure 5c shows the mean energy of protons within geosynchronous orbit (total energy divided by the number of particles). There is a sharp drop in mean energy after the first reconnection. This is explained in the following way: during the quiet period of northward $B_{z}$, before the IMF turned southward at 04:05, only very high energy particles populate the ring current, because lower energy particles experience much stronger charge exchange losses due to interaction with neutral particles [Fok et al., 1993]. This statement is confirmed by Figure 6, showing spectra of particles for two different moments of time: before and after the first reconnection occurred at 04:52. The dotted line in Figure 6 represents the spectrum of particles at the moment of time $\mathrm{t} 1=04: 15$, correspondent to the high, presubstorm level of the mean energy. This spectrum clearly demonstrates the domination of higher energies over lower energies in the presubstorm particle distribution. The solid line in Figure 6 shows the spectrum of particles at the moment of time $\mathrm{t} 2=05: 30$, correspondent to the postsubstorm, lower level of the mean energy. This time lower energy particles prevail in the postsubstorm particle distribution in correspondence to Figure 5c. Therefore we can conclude that the mean energy drops sharply as newly incoming particles have lower energy on the average. As convection and reconnection further energize ions, the ring current energy grows again slowly.

[22] Figure 7 represents particle number (Figure 7a) and energy (Figure 7b) gain and loss through FRC model boundaries. Here are shown time-integrated functions, so they do not demonstrate strong oscillations over the simulation period. It can be seen that more particles are injected through the nightside boundary than lost through the dayside boundary (Figure 7a), but energy content is balanced (Figure 7b). This means that more lower-energy particles are injected from the nighside boundary and fewer higher-energy particles are exited from the dayside boundary during the simulations.

[23] To analyze the spatial energy distribution of the ring current particles, we calculated the energy content within $L$ shell, produced by FRC model. The result for is shown in Figure 8 (the inner boundary of the FRC model is $\mathrm{L}=2$ and the outer is close to $\mathrm{L}=8$ ). It is clear that energy in the outer shells experience much stronger oscillations than energy in the inner shells. Thus the multiple reconnection influences outer regions stronger than the inner regions of the ring current.

[24] The large fluctuation in ring current energy at high Ls is also evident from Figure 9. Here Figure 9a shows the total proton energy content within geosynchronous orbit $\mathrm{R}=6.6 R_{E}$ and Figure $9 \mathrm{~b}$ shows the same for $\mathrm{R}=10 R_{E}$. Marked by the dotted line in these graphs are the correspondent energies for steady reconnection (storm), without taking into account of the nongyrotropy effects, which exhibit no oscillations at all. Figure 9a clearly shows also that the energy in the ring current is higher for the quasi periodic loading-unloading than for the steady reconnection.

[25] The higher level of the ring current energy oscillations for the outer regions demonstrated in the previous two figures is in agreement with the spatial distribution of the magnetic field variations. Figure 10 demonstrates depen-

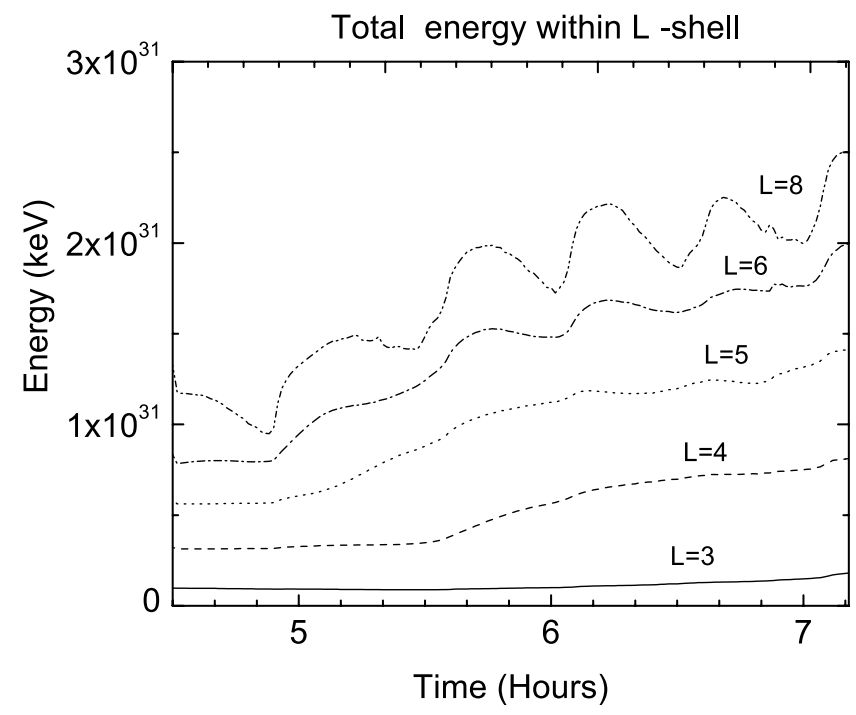

Figure 8. The energy content in $L$ shell given by the FRC model for $\mathrm{L}=3$ to $\mathrm{L}=8$ as a function of time. 

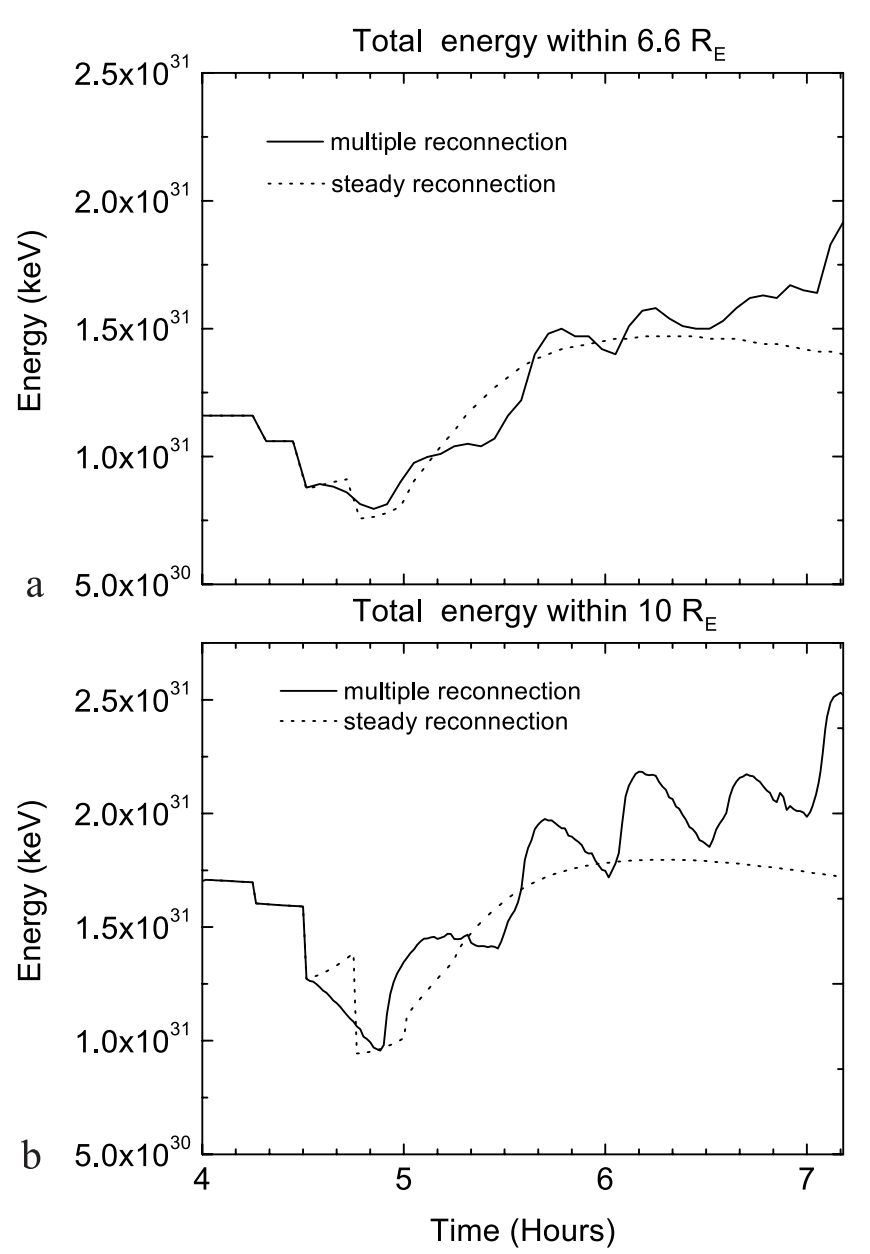

Figure 9. Proton energy content (a) within geosynchronous orbit $R=6.6 R_{E}$ and (b) within $R=10 R_{E}$ calculated through the FRC model as a function of time. Solid lines represent the multiple reconnection case due to nongyrotropy effects. Dotted lines correspond to the case of steady reconnection, when no gyrotropy effect was taken into account.

dence of the $B_{z}$ magnetic field component variations, $\delta B_{z}=$ $B_{z}(t)-B_{z}(t-\delta t)$ on different radial distances from the Earth. The variations are stronger for the outer regions $(R=$ $8 R_{E}$ ). Here we present the strongest variations, for magnetic local midnight, MLT $=00: 00$ only, because they are most significant to injection and energy oscillation. The flank and noon region variations (not shown) are usually much smaller.

\section{Summary}

[26] Numerical resistivity alone produces steady magnetosphere for steady solar wind conditions and periodical flipping of the IMF $B_{z}$ component with corresponding cross polar cap potential variation was required to reproduce geosynchronous flux oscillations in previous simulations [Keller et al., 2005]. Our model calculations demonstrate that proton fluxes in the ring current experience strong quasi-periodic oscillations even for steady southward
IMF $B_{z}$ component: the result never obtained before in simulations. This happens due to quasi-periodical loadingunloading process in the magnetotail when kinetic effects are taken into account in the reconnection region. In this case bursty inductive electric fields due to magnetic field variations cause quasi-periodical injection of particles into the ring current and corresponding geosynchronous flux oscillations.

[27] The total proton energy within geosynchronous orbit exhibits overall growth in time due to quasi-steady convection and oscillates due to injection through inductive electric field caused by multiple dipolarization. This combined effect of quasi-steady convective and bursty inductive electric fields is in agreement with observations [Reeves et al., 2004]. Simulations by Keller et al. [2005] demonstrated no overall growth of the ring current energy due to IMF $B_{z}$ flipping/cross polar cap potential variations. Similarly, the high level of the number of particles within geosynchronous orbit after the first reconnection event is maintained in our simulations by quasi-steady convection for steady southward IMF $B_{z}$ component. Keller et al. [2005] obtained no overall growth of the number of particles within geosynchronous orbit due to IMF $B_{z}$ flipping/cross polar cap potential variations. The detailed comparison of the ring current energization due to the two different mechanisms, (1) IMF $B_{z}$ flipping/cross polar cap potential variations and (2) quasi-periodic loading-unloading in the magnetotail, will be discussed in a separate publication.

[28] Calculated geosynchronous fluxes are almost dispersionless for local magnetic midnight and exhibit increasing energy dispersion, with more energetic particles coming first, followed by lower energy particles when going in westward direction. This happens due to energy dependence of particle gradient/curvature drift velocity in the ring current.

[29] The flux oscillation amplitude is stronger in the outer regions of the ring current although the regions close to the

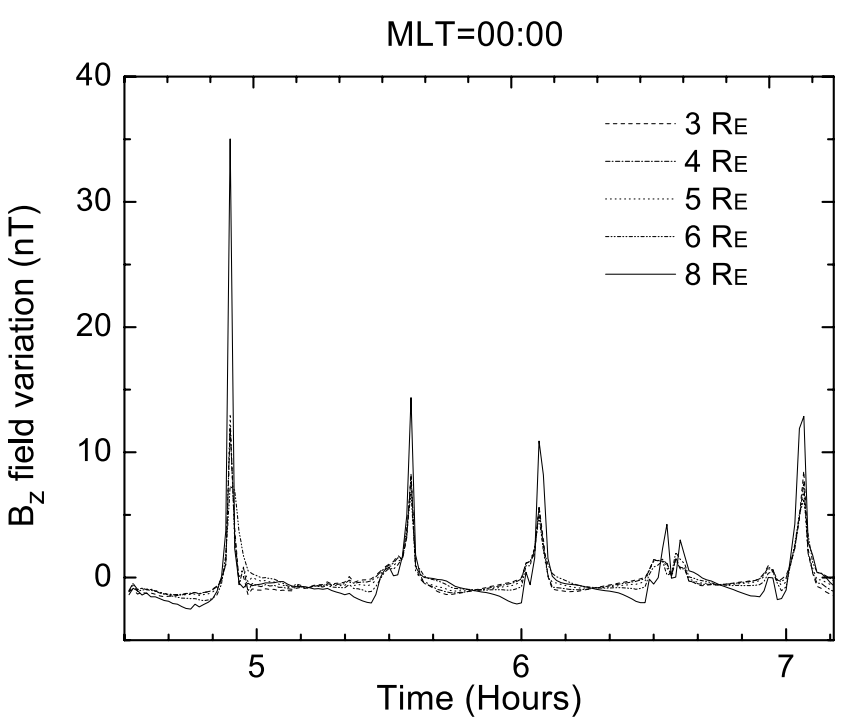

Figure 10. The variation of the magnetotail $B_{z}$ field component $\delta B_{z}=B_{z}(t)-B_{z}(t-\delta t)$ given by the BATS-RUS code as a function of time for different radial distances at MLT $=00: 00$. 
geosynchronous orbit experience substantial perturbations as well. This is in agreement with the spatial distribution of the magnetic field variations in the ring current region.

[30] Geosynchronous fluxes obtained in the presented work have many features of the "sawtooth" oscillations. However, the characteristic time of the tail loading-unloading process and hence of flux oscillations obtained in this work ( $230 \mathrm{~min})$ is shorter than usually observed "sawtooth" oscillation characteristic time ( $2-4$ hours). This disagreement may be associated with a number of additional factors, including conditions in the solar wind and in the inner magnetosphere and ionosphere, that are missing in the simulation with idealized symmetric settings, used by Kuznetsova et al. [2007]. The further improvement includes the use of less diffusive numerical schemes, higher-resolution simulation grids, improved representation of inner magnetosphere physics and magnetospheric convection. This work is under progress. In this paper we wanted to emphasize the importance of the incorporation of kinetic effects into global model and demonstrate the fact that reproduction of fast kinetic reconnection rates allowed modeling of "sawtooth" type geosynchronous flux oscillations.

[31] Finally, we showed that magnetic field variation in the magnetotail, not necessarily cross polar cap potential variations, are important for the geosynchronous energetic particle fluxes. The study with detailed comparison of the influence of these two effects on the ring current dynamics and energization is subject of separate future publication.

[32] Acknowledgments. We wish to thank J. Borovsky and G. Reeves for helpful discussions. This work was performed while one of the authors (A. T.) held a NRC/ORAU Research Associateship Award at Goddard Space Flight Center. Computations were performed at the Community Coordinated Modeling Center through the runs-on-request system.

[33] Amitava Bhattacharjee thanks Michael Denton and another reviewer for their assistance in evaluating this paper.

\section{References}

Birn, J., M. Thomsen, and M. Hesse (2004a), Electron acceleration in the dynamic magnetotail, Phys. Plasmas, 11, 1825-1833.

Birn, J., M. Thomsen, and M. Hesse (2004b), Acceleration of oxygen ions in the dynamic magnetotail: Test particle orbits in three-dimensional magnetohydrodynamic simulation fields, Ann. Geophys., 22, $1305-$ 1315 .

Daglis, I. A., R. M. Thorne, W. Baumjohann, and S. Orsini (1999), The terrestrial ring current: Origin, formation, and decay, Rev. Geophys., 37, $407-438$.

Dessler, A. J., and E. N. Parker (1959), Hydromagnetic theory of geomagnetic storms, J. Geophys. Res., 64, 2239-2252.

Ebihara, Y., and M. Ejiri (2000), Simulation study on fundamental properties of the storm-time ring current, J. Geophys. Res., 105, 15,84315,860 .
Ebihara, Y., M.-C. Fok, R. A. Wolf, M. F. Thomsen, and T. E. Moore (2005), Nonlinear impact of plasma sheet density on the storm-time ring current, J. Geophys. Res., 110, A02208, doi:10.1029/2004JA010435.

Fok, M.-C., and T. E. Moore (1997), Ring current modeling in a realistic magnetic field configuration, Geophys. Res. Lett., 24, 1775-1778.

Fok, M.-C., J. U. Kozyra, A. F. Nagy, C. E. Rasmussen, and G. V. Khazanov (1993), Decay of equatorial ring current ions and associated aeronomical consequences, J. Geophys. Res., 98, 19,381-19,394.

Fok, M.-C., T. E. Moore, and M. E. Greenspan (1996), Ring current development during storm main phase, J. Geophys. Res., 101, 15,31115,322 .

Fok, M.-C., T. E. Moore, and D. C. Delcourt (1999), Modeling of inner plasma sheet and ring current during substorms, J. Geophys. Res., 104, $14,557-14,569$.

Fok, M.-C., R. A. Wolf, R. W. Spiro, and T. E. Moore (2001), Comprehensive computational model of Earth's ring, J. Geophys. Res., 106, $8417-8421$.

Henderson, M. G., G. D. Reeves, R. Skoug, M. F. Thomsen, M. H. Denton, S. B. Mende, T. J. Immel, P. C. Brandt, and H. J. Singer (2006a), Magnetospheric and auroral activity during the 18 April 2002 sawtooth event, J. Geophys. Res., 111, A01S90, doi:10.1029/2005JA011111.

Henderson, M. G., et al. (2006b), Substorms during the 10-11 August 2000 sawtooth event, J. Geophys. Res., 111, A06206, doi:10.1029/ 2005JA011366.

Keller, K. A., M.-C. Fok, A. Narock, M. Hesse, L. Rastaetter, M. M. Kuznetsova, T. I. Gombosi, and D. L. DeZeeuw (2005), Effect of multiple substorms on the buildup of the ring current, J. Geophys. Res., 110, A08202, doi:10.1029/2004JA010747.

Kuznetsova, M., M. Hesse, L. Rastaetter, G. Toth, D. DeZeeuw, and T. Gombosi (2006), Multi-scale modeling of magnetospheric reconnection, Eos Trans. $A G U, 87(37)$, Jt. Assem. Suppl., Abstract SM21A-04.

Kuznetsova, M. M., M. Hesse, L. Rastatter, A. Taktakishvili, G. Toth, D. L. De Zeeuw, A. Ridley, and T. I. Gombosi (2007), Multiscale modeling of magnetospheric reconnection, J. Geophys. Res., doi:10.1029/2007JA012316, in press.

Powell, K. G., P. L. Roe, T. J. Linde, T. I. Gombosi, and D. L. DeZeeuw (1999), A solution-adaptive upwind scheme for ideal magnetohydrodynamics, J. Comput. Phys., 154, 284-309.

Reeves, G. D., and M. G. Henderson (2001), The storm-substorm relationship: Ion injections in geosynchronous measurements and composite energetic neutral atom images, J. Geophys. Res., 106, 5833-5844.

Reeves, G. D., et al. (2004), IMAGE, POLAR, and geosynchronous observations of substorm and ring current ion injections, in Disturbances in Geospace: The Storm-Substorm Relationship, Geophys. Monogr. Ser. vol. 142, edited by A. S. Sharma, Y. Kamide, and G. S. Lakhina, pp. 91-101, AGU, Washington, D. C.

Sheldon, R. B., and D. C. Hamilton (1993), Ion transport and loss in the Earth's quiet ring current: 1. Data and standard model, J. Geophys. Res., 98, 13,491-13,508.

Wolf, R. A., and M. Harel (1979), Dynamics of the magnetospheric plasma, in Dynamics of the Magnetosphere, edited by S.-I. Akosofu, pp. 143163, Springer, New York.

Wolf, R. A., J. W. Freeman Jr., B. A. Hausman, R. W. Spiro, R. V. Hilmer, and R. L. Lambour (1997), Modeling convection effects in magnetic storms, in Magnetic Storms, Geophys. Monogr. Ser., vol. 98, edited by B. T. Tsurutani et al., pp. 161-172, AGU, Washington, D. C.

A. Chulaki, M.-C. Fok, M. Hesse, M. M. Kuznetsova, M. Maddox, L. Rastätter, and A. Taktakishvili, Space Weather Laboratory, NASA Goddard Space Flight Center, Code 674, Bld. 21, Rm. 227, Greenbelt, MD 20771,USA. (staktak@helio.gsfc.nasa.gov)

D. L. De Zeeuw and T. I. Gombosi, Center for Space Environment Modeling, University of Michigan, Ann Arbor, MI 48109-2143, USA. 